\title{
Three-Year Tracking of Fatty Acid Composition of Plasma Phospholipids in Healthy Children
}

\author{
António Guerra $^{a} \quad$ Hans Demmelmair $^{b} \quad$ André Michael Toschke $^{c}$ Berthold Koletzko ${ }^{b}$ \\ ${ }^{a}$ Department of Paediatrics, H.S. João Faculty of Medicine, University of Porto, Porto, Portugal; ${ }^{b}$ Division of \\ Metabolic Diseases and Nutrition, Dr. von Haunersches Kinderspital, and ' Institute of Social Pediatrics and \\ Adolescent Medicine, Division of Epidemiology, University of Munich, Munich, Germany
}

\section{Key Words \\ Phospholipid fatty acids • Dietary fat · Tracking of fatty acid composition - Plasma phospholipid fatty acid composition, children}

\begin{abstract}
Objectives: The fatty acid composition of plasma phospholipids reflects the dietary fatty acid intake as well as endogenous turnover. We aimed at investigating the potential tracking of plasma phospholipid fatty acid composition in children that participated in a prospective cohort study. Methods: 26 healthy children participated in a longitudinal study on health risks and had been enrolled after birth. All children were born at term with birth weights appropriate for gestational age. Follow-up took place at ages 24, 36 and 60 months. At each time point a 24-hour dietary recall was obtained, anthropometric parameters were measured and a blood sample for phospholipid fatty acid analysis was taken. Results: Dietary intake of saturated (SFA), monounsaturated (MUFA) and polyunsaturated (PUFA) fatty acids at the three time points were not correlated. We found lower values for plasma MUFA and the MUFA/SFA ratio at 60 months compared to 24 months. In contrast, total PUFA, total $n-6$ and n-6 long-chain polyunsaturated fatty acids (LC-PUFA) were higher at 60 months. Significant averaged correlation coefficients (average of Pearson's R for 24 versus 36 months and 36 versus 60 months) were found for $n-6$ LC-PUFA ( $r=0.67)$,
\end{abstract}

\section{KARGER}

Fax +41613061234

E-Mail karger@karger.ch

www.karger.com

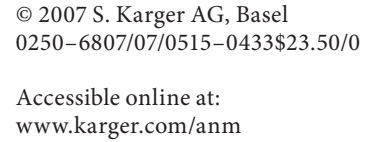

$n-6 / n-3$ LC-PUFA ratio $(r=0.59)$ and arachidonic acid/linoleic acid ratio $(r=0.64)$. Partial tracking was found for the docosahexaenoic acid $/ \alpha$-linolenic acid ratio $(r=0.33)$. Body mass index and sum of skinfolds Z-scores were similar in the three evaluations. Conclusions: A significant tracking of $n-6$ LC-PUFA, $n-6$ LC-PUFA/n-3 LC-PUFA ratio, arachidonic acid/ linoleic acid ratio and docosahexaenoic acid $/ \alpha$-linolenic acid ratio may reflect an influence of individual endogenous fatty acid metabolism on plasma concentrations of some, but not all, fatty acids.

Copyright $\odot 2007$ S. Karger AG, Basel

\section{Introduction}

Cardiovascular disease is the main cause of morbidity and mortality among adults in industrialized countries [1]. It is unanimously recognized that atherosclerosis originates in childhood although clinical expressions of cardiovascular disease only appear during adulthood [27]. Quality and quantity of dietary fat are an important environmental factor influencing serum lipid profile and hence susceptibility to atherosclerosis. Fatty acid composition of plasma lipid classes reflects food habits and is associated with the risk of coronary heart disease [8]. Plasma phospholipid fatty acid composition is accepted as a reliable biomarker of dietary fatty acid intake in observational and interventional studies [9-11]. Due to a 
slower turnover of plasma phospholipids than plasma triacylglycerols, day-to-day variation is attenuated, but on the other hand, almost instant incorporation of dietary fatty acids has been demonstrated in tracer studies [12]. However, in addition to dietary fat intake, endogenous fatty acid and phospholipid metabolism modulates plasma and membrane fatty acid composition [13-15].

Plasma fatty acid composition of children was determined at three time points during pre-school age to assess the extension of fatty acid tracking over time, with the aim to distinguish fatty acids or fatty acid classes according to the degree of metabolic control of their respective plasma concentrations.

\section{Subjects and Methods}

Twenty-six children (13 male, 13 female) participated in a longitudinal investigation on health risks and had been enrolled after birth. All children were born at term with birth weights appropriate for gestational age. Blood samples were obtained at age 24, 36 and 60 months in combination with scheduled preventive health checks.

The study was approved by the local ethical committees, and informed parental consent was obtained for each subject before enrolment.

Venous blood samples were obtained from an antecubital vein with sodium ethylene diamine tetraacetate $(1 \mathrm{mg} / \mathrm{ml})$ as anticoagulant. After centrifugation, plasma was immediately frozen in plastic vials with a snap-on lid and stored at $-18^{\circ} \mathrm{C}$ until analysis. Plasma lipids were extracted into chloroform/methanol (2/1) [16]. The phospholipid fraction was isolated by thin-layer chromatography. Fatty acid methyl esters were prepared by transesterification with methanolic $\mathrm{HCl}$ and analyzed by high-resolution capillary gas chromatography as described previously [17]. Results were expressed as percentage (wt/wt) of all FA detected with a chain length between 12 and 24 carbon atoms.

Weight, height, tricipital and subscapular skin folds were measured [18] and body mass index was calculated [19]. Data from the National Center of Health Statistics was used as reference values [20]. Food habits were obtained via 24-hour recalls [21]. Eating models were used to enable easier collection of information and to quantify results [21]. The McCance and Widdowson's tables were used to convert food intake into energy and nutrient intake [22].

Z-scores of age-specific fatty acid levels were calculated based on mean levels and corresponding standard deviations (SD). Pearson correlation coefficients of age-specific Z-scores were estimated to assess level of tracking. The corresponding test of significance for Pearson correlation coefficients was not used due to its unhelpful null hypothesis $(\mathrm{H} 0: \rho=0)$. Overall tracking was assumed if Pearson correlation coefficients for 24 versus 60 and 36 versus 60 months exceeded 0.4. Partial (or later) tracking was assumed for Pearson correlation coefficients $\leq 0.4$ between 24 and 36 months, but Pearson correlation coefficients $>0.4$ between 36 and 60 months. Additionally, time period-weighted averages were calculated from correlation coefficients from 24 to 36 and 36 to
Table 1. Mean daily intake (SD) of fatty acid classes (\% energy of total fat-derived energy for those food items with available information for individual fatty acid classes) at age 24, 36 and 60 months $(\mathrm{n}=26)$

\begin{tabular}{lcrr}
\hline & 24 months & 36 months & 60 months \\
\hline SFA, \%* & $32.1(15.9)$ & $46.3(5.2)$ & $47.5(4.5)$ \\
MUFA, \%* & $56.9(14)$ & $46.2(3.8)$ & $44.2(3.3)$ \\
PUFA, \% & $11(10.2)$ & $7.6(2.3)$ & $8.3(2.2)$ \\
\hline
\end{tabular}

SFA = Saturated fatty acids; MUFA = monounsaturated fatty acids; PUFA = polyunsaturated fatty acids.

* Significant difference between time points $(\mathrm{p}<0.05)$, identified by ANOVA.

Table 2. Mean levels (SD) of Z-scores of body mass index and sum of tricipital and subscapular skinfolds at age 24, 36 and 60 months $(\mathrm{n}=26)$

\begin{tabular}{llll}
\hline Z-scores & 24 months & 36 months & 60 months \\
\hline Body mass index & $0.5(1.1)$ & $0.23(1.2)$ & $0.64(1.3)$ \\
Sum of skinfolds & $0.1(0.66)$ & $0.28(0.89)$ & $0.12(0.83)$ \\
\hline
\end{tabular}

No significant differences $(\mathrm{p}<0.05)$ between time points identified by ANOVA.

60 months. Differences of mean levels at time points were tested by analysis of variance.

All calculations were carried out with the software package SAS version 9.1 (SAS Institute Inc., Cary, N.C., USA) and with the statistical software package R2.0.1 (http://www.r-project.org/).

\section{Results}

\section{Dietary Fat Intake}

As expected, total daily energy intake of the infants increased with advancing age from $891 \pm 168 \mathrm{kcal}$ at 24 months to $1,331 \pm 309 \mathrm{kcal}$ at 36 months and to $1,572 \pm$ $358 \mathrm{kcal}$ at 60 months of age. Food habits changed from infancy to school age with an increasing percentage of energy from total fat $(29.6 \pm 6.4 \%$ at 24 months, $36.9 \pm$ $5.2 \%$ at 36 months, $38.9 \pm 5.6$ at 60 months). The contribution of saturated fatty acids (SFA) increased with advancing age, while monounsaturated fatty acids (MUFA) and PUFA decreased (table 1). Intake of SFA, MUFA and polyunsaturated fatty acids (PUFA, $\geq 18 \mathrm{C}$-atoms, $\geq 2$ double bonds) was not significantly correlated between the three time points. 
Table 3. Mean weight percentages (SD) of plasma phospholipid fatty acids at age 24 , 36 and 60 months $(n=26)$

\begin{tabular}{lccc}
\hline & 24 months & 36 months & 60 months \\
\hline SFA $^{*}$ & $50.2(2.1)$ & $47.3(1.2)$ & $48.5(1.3)$ \\
MUFA $^{*}$ & $13.6(2.1)$ & $12.5(1.5)$ & $11.1(1.5)$ \\
PUFA $^{*}$ & $35.6(2.9)$ & $39.7(1.6)$ & $39.9(1.8)$ \\
$n-6$ LC-PUFA & $11.8(1.7)$ & $14.2(1.6)$ & $13.8(1.7)$ \\
PUFA/SFA & $3.4(0.9)$ & $4.2(0.8)$ & $4.1(1.0)$ \\
MUFA/SFA* & $0.71(0.1)$ & $0.84(0.1)$ & $0.82(0.1)$ \\
$n-6$ LC-PUFA/n-3 LC-PUFA & $0.27(0.04)$ & $0.27(0.04)$ & $0.23(0.03)$ \\
AA/LA & $3.7 \pm 1.1$ & $3.5 \pm 0.9$ & $3.5 \pm 0.8$ \\
DHA/ALN & $0.39(0.1)$ & $0.47(0.1)$ & $0.44(0.1)$ \\
\hline
\end{tabular}

* ANOVA $\mathrm{p}<0.05$.

LC-PUFA = Long-chain polyunsaturated fatty acids; PUFA = polyunsaturated fatty acids; MUFA = monounsaturated fatty acids; SFA = saturated fatty acids; $\mathrm{AA}=$ arachidonic acid; $\mathrm{LA}=$ linoleic acid; $\mathrm{DHA}=$ docosahexaenoic acid; $\mathrm{ALN}=\alpha$-linolenic acid.

\begin{tabular}{|c|c|c|c|c|c|}
\hline & PUFA & $\begin{array}{l}n-6 \text { LC- } \\
\text { PUFA }\end{array}$ & $n-3$ LC PUFA & $\begin{array}{l}n-6 \text { LC-PUFA/ } \\
n-3 \text { LC-PUFA }\end{array}$ & $\begin{array}{l}\text { PUFA/ } \\
\text { SFA }\end{array}$ \\
\hline \multicolumn{6}{|l|}{ Energy intake } \\
\hline 24 months & $0.52^{* *}$ & 0.11 & 0.23 & -0.24 & $0.5^{*}$ \\
\hline 36 months & -0.1 & -0.1 & -0.41 & 0.36 & -0.16 \\
\hline 60 months & 0.02 & 0.35 & -0.01 & 0.2 & 0.1 \\
\hline \multicolumn{6}{|l|}{ Fat intake } \\
\hline 24 months & $0.53^{* *}$ & 0.06 & 0.15 & -0.21 & $0.53^{* *}$ \\
\hline 36 months & -0.05 & -0.2 & $-0.43^{*}$ & 0.35 & -0.15 \\
\hline 60 months & 0.23 & $0.52^{*}$ & 0.1 & 0.1 & 0.26 \\
\hline
\end{tabular}

${ }^{*} \mathrm{p}<0.05 ;{ }^{* *} \mathrm{p}<0.01$.
Table 4. Correlation between plasma fatty acids and dietary PUFA intake (\% of total energy intake and weight percent of fat intake) at age 24,36 and 60 months
Correlations of plasma phospholipid fatty acids be-

\section{Nutritional Status}

Body mass index and sum of tricipital and subscapular skinfolds of the children observed at the three time points were similar, indicating no change with time of the nutritional status (table 2).

\section{Fatty Acid Composition of Plasma Phospholipids}

Differences were found between plasma phospholipid fatty acids from the different sampling points. MUFA and SFA were lower at 60 months compared to 24 months, while total PUFA were higher at age 60 months (table 3).

A significant correlation between PUFA intake and plasma fatty acids was particularly observed at 24 months (table 4). Expressing PUFA intake as percentage of daily energy intake (kcal) or as percentage of daily fat intake (g) did not significantly influence the results.

Fatty Acid Composition of Plasma Phospholipids in Healthy Children tween the three time points are shown in table 5. The highest weighted average correlations between time points were observed for $n-6$ long-chain polyunsaturated fatty acids (LC-PUFA, $\geq 20$ C-atoms, $\geq 2$ double bonds), for the $n-6 / n-3$ LC-PUFA ratio (fig. 1 ) and for the arachidonic acid/linoleic acid ratio. Partial tracking was found for the docosahexaenoic acid/ $\alpha$-linolenic acid ratio (table 5). No tracking was observed for SFA, MUFA or total PUFA.

\section{Discussion}

In the children studied, dietary intake changed from 2 years to school age, with an increasing percentage of energy from total fat which was similarly contributed by

Ann Nutr Metab 2007;51:433-438 
Table 5. Pearson correlation coefficients between age-specific Z-scores of plasma phospholipid fatty acids at different time points

\begin{tabular}{lllll}
\hline \multirow{2}{*}{ Fatty acid } & \multicolumn{4}{l}{ Pearson correlation coefficient } \\
\cline { 2 - 5 } & $\begin{array}{l}24 \text { vs. } 36 \\
\text { months }\end{array}$ & $\begin{array}{l}24 \text { vs. } 60 \\
\text { months }\end{array}$ & $\begin{array}{l}36 \text { vs. } 60 \\
\text { months }\end{array}$ & $\begin{array}{l}\text { weighted } \\
\text { average }\end{array}$ \\
\hline SFA & 0.00 & -0.02 & 0.13 & 0.09 \\
MUFA & 0.40 & 0.25 & 0.71 & 0.61 \\
PUFA & 0.43 & 0.14 & 0.47 & 0.46 \\
$n-6$ LC-PUFA & 0.68 & 0.63 & 0.67 & 0.67 \\
$n-3$ LC-PUFA & 0.21 & 0.31 & 0.38 & 0.32 \\
PUFA/SFA & 0.30 & 0.04 & 0.23 & 0.25 \\
MUFA/SFA & 0.28 & 0.20 & 0.67 & 0.54 \\
$n-6$ LC-PUFA/n-3 LC-PUFA & 0.48 & 0.58 & 0.64 & 0.59 \\
AA/LA & 0.77 & 0.63 & 0.57 & 0.64 \\
DHA/ALN & 0.13 & 0.47 & 0.43 & 0.33 \\
\hline
\end{tabular}

LC-PUFA = Long-chain polyunsaturated fatty acids; PUFA = polyunsaturated fatty acids; $\mathrm{MUFA}=$ monounsaturated fatty acids; SFA = saturated fatty acids; $\mathrm{AA}=$ arachidonic acid; LA = linoleic acid; DHA = docosahexaenoic acid; $\mathrm{ALN}=\alpha$-linolenic acid.

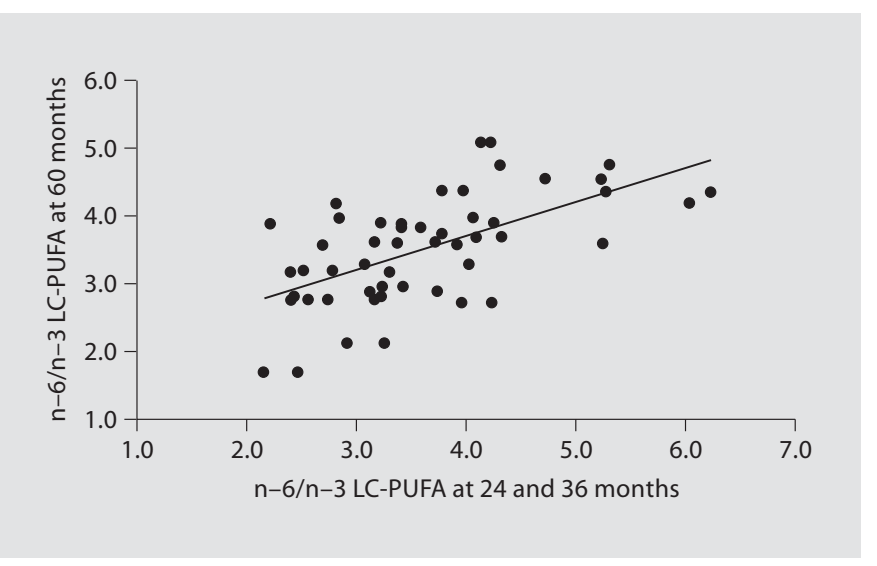

Fig. 1. Overall tracking ( 24 vs. 60 and 36 vs. 60 months) for plasma phospholipid $n-6 / n-3$ long-chain polyunsaturated fatty acids ratio $(\mathrm{r}=0.59)$.

all major groups of fatty acids (table 1). The increasing percentage of energy from SFA might be due to an increasing consumption of animal fat (milk, butter and lard) at age 60 months. Intake of SFA, MUFA and PUFA were not correlated between the three time points agreeing with intraindividual changes in food preferences but also with large day-to-day variation.

There is an ongoing discussion about the most suitable compartment to sample for the analysis of a biomarker for fatty acid intake [23]. Plasma triacylglycerols, cholesterol esters and phospholipids have been shown to be indicative for dietary compliance in clinical studies, al- though compositional changes are generally not proportional to dietary changes [24]. As for none of these lipid fractions the period for which they indicate the dietary fatty acid composition can exactly be specified and as we were mainly interested in arachidonic and docosahexaenoic acids, we decided to analyze plasma phospholipids, which show relatively high percentages of these fatty acids. Furthermore, a very good correlation for PUFA percentages in muscle phospholipids and serum phospholipids has been observed ( $r=0.8$ for both arachidonic and docosahexaenoic acid) in study participants on controlled diets for 3 months [25]. Thus, plasma phospholipids can be indicative of whole-body PUFA status under steadystate conditions.

In our subjects a correlation between PUFA from diet and plasma PUFA and PUFA/SFA could be observed at age 24 months but not at the older ages (table 4). SFA and MUFA from diet did not correlate with plasma fatty acids at any of the three time points. Similar results were observed in other studies in children older than 9 years and in adults [26].

This might be explained by a more stable food pattern at age 2 years and a greater day-to-day variability of food intake in older children. Furthermore, 24-hour recall is not a very accurate method for the assessment of dietary intake $[27,28]$. High variability in nutrient intakes requires prolonged dietary recording of food habits in order to obtain precise and reliable information [29]. Unfortunately, under the given study conditions, this was not possible as compliance with 3-day food records was very poor. Nevertheless, the absence of a correlation be- 
tween the observed dietary intakes at the different infantile ages studied and no correlation between PUFA intake and plasma phospholipid PUFA content is in agreement with an important further influencing factor on plasma PUFA, which might be endogenous metabolism.

The food recall was performed on the day preceding the collection of blood samples. Changes in phospholipid fatty acid composition occur only within 1 or 2 weeks [30], thus a high correlation of the results cannot be expected. Although metabolism seems to influence fatty acid composition of plasma phospholipids and cholesterol esters in addition to dietary intake, they might reflect medium-term intake of fatty acids better than many of the questionnaire based methods applied for dietary evaluation [31].

Although dietary PUFA were not correlated at the three time points, $n-6$ LC-PUFA and $n-6 / n-3$ LC-PUFA ratio in plasma phospholipids were correlated between the three observations with weighted average correlation coefficients of 0.67 and 0.59 respectively, while $n-3$-LCPUFA did not track between time points (table 5). A low and irregular intake of fish in the studied population may explain these results. However, the $n-6 / n-3$ LC-PUFA ratio, which seems important for a balanced synthesis of eicosanoids, correlated between the three time points. Although less pronounced compared to the arachidonic acid/linoleic acid ratio, a tracking of the docosahexaenoic acid $/ \alpha$-linolenic acid ratio was also observed (table 5; fig. 1).

The tracking of the plasma fatty acid percentages over time observed in our study agrees in principle with observations in groups of Finnish children and adolescents in respect to LC-PUFA, as higher tracking was described for arachidonic acid $(\mathrm{r}=0.54)$ than for docosahexaenoic acid $(r=0.38)$ over 6 years [32]. The fact that in this study ad- ditionally lower but significant tracking for individual saturated and monounsaturated fatty acids was observed, might be explained by the higher number of subjects studied and the observation that tracking of individual fatty acids is higher than tracking of groups of fatty acids [33].

The tracking of $n-6$ LC-PUFA percentages is in agreement with a significant influence of individual endogenous PUFA metabolism on plasma phospholipid composition in children of this age group [34]. This corresponds to the demonstration of a clear influence of genetic variants of the desaturation system on the plasma phospholipid n-6 LC-PUFA percentage in adults, while $n-3$ LCPUFA were much less affected by these genes [35]. As LCPUFA are relevant modulators of membranes properties [36], are required as precursors for the synthesis of biologically active substances, e.g. eicosanoids $[37,38]$, and are involved in the regulation of gene expression [39], this might be of considerable importance.

We did not find significant tracking of plasma phospholipid SFA and MUFA between time points, which indicates less importance of the endogenous metabolism.

In conclusion, the present study shows that plasma phospholipid fatty acid composition has a overall tracking for $n-6$ LC-PUFA, $n-6 / n-3$ LC-PUFA ratio and arachidonic acid/linoleic acid ratio, and a partial (or later) tracking for docosahexaenoic acid/ $\alpha$-linolenic acid ratio which might be related to interindividual differences of the activity of the desaturation/elongation pathway. This points towards the importance of personalized dietary recommendations.

\section{Acknowledgement}

B.K. is the recipient of a Freedom to Discover Award of the Bristol Myers Squibb Foundation, New York, N.Y., USA.

\section{References}

1 World Health Organization: World Health Statistics Annual 1991. Geneva, WHO, 1992.

2 Kwiterovich PO Jr: Biochemical, clinical, epidemiologic, genetic and pathologic data in the pediatric age group relevant to the cholesterol hypothesis. Pediatrics 1986;78:349362.

3 National Cholesterol Education Program (NCEP): Highlights of the report of the expert panel on blood cholesterol levels in children and adolescents. Pediatrics 1992;89: 495-501.
4 Berenson GS, McMahan CA, Voors AW: Cardiovascular Risk Factors in Children The Early Natural History of Atherosclerosis and Essential Hypertension. New York, Oxford University Press, 1980.

5 Vobecky JS, Grant AM, Laplante P, et al: Hypercholesterolaemia in childhood: repercussions in adulthood. Eur J Clin Nutr 1993; 47(suppl 1):S47-S56.

6 Lenfant C, Savage PJ: The early natural history of atherosclerosis and hypertension in the young: National Institutes of Health Perspectives. Am J Med Sci 1995;310(suppl 1): S3-S7.
7 Berenson GS, Wattigney WA, Bao W, et al: Rationale to study the early natural history of heart disease: The Bogalusa Heart Study. Am J Med Sci 1995;310(suppl 1):S22-S28.

8 Miettinen TA, Naukkarinen V, Huttunen JK, et al: Fatty acid composition of serum lipids predicts myocardial infarction. Br Med J 1982;285:993-996.

9 Svensson BG, Akesson B, Nilsson A, et al: Fatty acid composition of serum phosphatidylcholine in healthy subjects consuming varying amounts of fish. Eur J Clin Nutr 1993;47:132-140. 
10 Ma J, Folsom AR, Shahar E, Eckfeldt JH: Plasma fatty acid composition as an indicator of habitual dietary fat intake in middleaged adults. The Atherosclerosis Risk in Communities (ARIC) Study Investigators. Am J Clin Nutr 1995;62:564-571.

11 Di Stasi D, Bernasconi R, Marchioli R, Marfisi RM, Rossi G, Tognoni G, et al: Early modifications of fatty acid composition in plasma phospholipids, platelets and mononucleates of healthy volunteers after low doses of $n-3$ polyunsaturated fatty acids. Eur J Clin Pharmacol 2004;60:183-190.

12 Demmelmair H, Iser B, Rauh-Pfeiffer A, Koletzko B: Comparison of bolus versus fractionated oral applications of $\left[{ }^{13} \mathrm{C}\right]$-linoleic acid in humans. Eur J Clin Invest 1999;29: 603-609.

13 Koletzko B, Decsi T, Demmelmair H: Arachidonic acid supply and metabolism in human infants born at full term. Lipids 1996;31 79-83.

14 Luukkainen P, Salo MK, Janas M, et al: Fatty acid composition of plasma and red blood cell phospholipids in preterm infants from 2 weeks to 6 months postpartum. J Pediatr Gastroenterol Nutr 1995;20:310-315.

15 Vance JE, Vance DE: Phospholipid biosynthesis in mammalian cells. Biochem Cell Biol 2004;82:113-128.

16 Folch J, Lees M, Stanley GHS: A simple method for the isolation and purification of total lipids from animal tissues. J Biol Chem 1957;226:497-509.

17 Decsi T, Koletzko B: Fatty acid composition of plasma lipid classes in healthy subjects from birth to young adulthood. Eur J Pediatr 1994; 153:520-525.

18 Jelliffe DB, Jelliffe EFP: Direct assessment of nutritional status. Anthropometry: major measurements; in Jelliffe DB, Jelliffe EFP (eds): Community Nutritional Assessment with Special Reference to Less Technically Developed Countries. New York, Oxford University Press, 1989, pp 68-105.
19 Lee J, Kolonel LN, Hinds MW: Relative merits of the weight-corrected-for-height indices. Am J Clin Nutr 1981;34:2521-2529.

20 Hamill PV, Drizd TA, Johnson CL, et al: Physical growth: National Center for Health Statistics percentiles. Am J Clin Nutr 1979; 32:607-629.

21 Frank GC, Berenson GS, Schilling PE, et al: Adapting the 24-hour recall for epidemiologic studies of schoolchildren. J Am Diet Assoc 1977;71:26-31.

22 Paul AA, Southgate DAT: McCance and Widdowson's. The Composition of Foods, ed 3. London, HMSO, 1985.

23 Baylin A, Kim MK, Donovan-Palmer A, et al: Fasting whole blood as a biomarker of essential fatty acid intake in epidemiologic studies: comparison with adipose tissue and plasma. Am J Epidemiol 2005; 162:373-381.

24 Baylin A, Campos H: The use of fatty acid biomarkers to reflect dietary intake. Curr Opin Lipidol 2006;17:22-27.

25 Andersson A, Nalsen C, Tengblad S, Vessby B: Fatty acid composition of skeletal muscle reflects dietary fat composition in humans. Am J Clin Nutr 2002;76:1222-1229.

26 Moilanen T, Rasanen L, Viikari J, et al: Correlation of serum fatty acid composition with dietary intake data in children and young adults. Ann Med 1992;24:67-70.

27 Serdula MK, Alexander MP, Scanlon KS, et al: What are preschool children eating? A review of dietary assessment. Annu Rev Nutr 2001;21:475-498.

28 Rockett HR, Colditz GA: Assessing diets of children and adolescents. Am J Clin Nutr 1997;65(suppl 4):1116S-1122S.

29 Livingstone MB, Robson PJ: Measurement of dietary intake in children. Proc Nutr Soc 2000;59:279-293.
30 Katan MB, Grundy SM, Willett WC: Should a low-fat, high-carbohydrate diet be recommended for everyone? Beyond low-fat diets. N Engl J Med 1997;337:563-566.

31 Riboli E, Ronnholm H, Saracci R: Biological markers of diet. Cancer Surv 1987;6:685718

32 Moilanen T, Rasanen L, Viikari J, Akerblom HK, Nikkari T: Tracking of serum fatty acid composition: a 6-year follow-up study in Finnish youths. Am J Epidemiol 1992;136: 1487-1492.

33 Zeleniuch-Jacquotte A, Chajes V, Van Kappel AL, Riboli E, Toniolo P: Reliability of fatty acid composition in human serum phospholipids. Eur J Clin Nutr 2000;54:367-372.

34 Tang C, Cho HP, Nakamura MT, Clarke SD: Regulation of human $\Delta 6$-desaturase gene transcription: identification of a functional direct repeat-1 element. J Lipid Res 2003;44: 686-695.

35 Schaeffer L, Gohlke H, Muller M, Heid IM, Palmer LJ, Kompauer I, et al: Common genetic variants of the FADS1 FADS2 gene cluster and their reconstructed haplotypes are associated with the fatty acid composition in phospholipids. Hum Mol Genet 2006; 15:1745-1756.

36 Youdim KA, Martin A, Joseph JA: Essential fatty acids and the brain: possible health implications. Int J Dev Neurosci 2000;18:383399.

37 Sellmayer A, Koletzko B: Long-chain polyunsaturated fatty acids and eicosanoids in infants - physiological and pathophysiological aspects and open questions. Lipids 1999; 34:199-205.

38 Koletzko B, Rodriguez-Palmero MJ: Polyunsaturated fatty acids in human milk and their role in early infant development. J Mammary Gland Biol Neoplasia 1999;4: 269-284.

39 Ntambi JM, Bene H: Polyunsaturated fatty acid regulation of gene expression. J Mol Neurosci 2001;16:273-278. 